\title{
Mitteilungen des Berufsverbands der Pneumologen in Baden-Württemberg
}

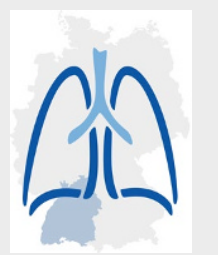

\section{Geschäftsstelle}

c/o med info $\mathrm{GmbH}$

Hainenbachstraße 25,

89522 Heidenheim

Tel 07321 9469182, Fax 073219469140

info@pneumologenverband.de, ww.pneumo-bw.de
Dr. Frank J. Heimann

(1. Vorsitzender, V.i.S.d.P.)

Dr. Michael Barczok (2. Vorsitzender)

Dr. Konrad Pumpe (Schatzmeister)

Dr. Stefan Veitshans (Schriftführer)
Dr. Erhard Bode (1. Beisitzer)

Dr. Andreas Hupert (2. Beisitzer)

Dr. Thushira Weerawarna

(Fortbildungsbeauftragter)

\section{QZ goes Online}

Getriggert durch die aktuellen Rahmenbedingungen wird auch in der Medizin die Digitalisierung und Webinarisierung rascher vorangetrieben als von vielen Akteuren erwartet. So fand auch der Qualitätszirkel der Pneumologen in Nordwürttemberg (QZPNW) im Juni erstmalig und erfolgreich als Online-Qualitätszirkel statt und kann als Modell für zukünftige Qualitätszirkel dienen.

Der QZPNW findet schon seit Jahren dreimal jährlich als Präsenzveranstaltung in Stuttgart statt. Neben Fachreferaten zu den unterschiedlichen pneumologischen Themenfeldern ist in der zweiten Hälfte der Veranstaltung die Besprechung der aktuellen berufspolitischen Themen fester Bestandteil. Die Teilnehmerzahlen liegen in der Regel zwischen 25 und 35 Pneumolog ${ }^{\star}$ Innen aus Niederlassung und Klinik; wobei es, bedingt durch die weiten Anfahrtswege aus den entfernteren Landkreisen, meistens die Kolleg^Innen aus den benachbarten Landkreisen sind, die teilnehmen. Damit erreichen sowohl die fachlichen als auch die berufspolitischen Themen jeweils nur einen kleinen Teil der Kolleg*Innen.

Die QZ-Region Nordwürttemberg umfasst im südlichen Bereich die Landkreise Böblingen, Esslingen, Göppingen, Heidenheim, Ludwigsburg, Stuttgart, Rems-Murr-Kreis, Ostalbkreis, im nördlichen Bereich Heilbronn, Hohenlohekreis, Schwäbisch Hall und Main-Tauber-Kreis. Anfahrtswege nach
Stuttgart sind z. B. von Bad Mergentheim $115 \mathrm{~km}$ und von Aalen rund $80 \mathrm{~km}$ bzw. laut google maps (ohne Stau und Berufsverkehr) 1 h 20 min bzw. 1 h.

Für Juni war geplant, den QZ in der Nähe von Heilbronn stattfinden zu lassen, um Kolleg*Innen aus den nördlicheren Regionen der QZ-Region kürzere Wege zu ermöglichen und den Kreis der üblichen Teilnehmer^Innen zu erweitern. Absprachen zu Fahrgemeinschaften aus den südlichen Kreisen waren bereits getroffen als klar wurde, dass der QZ coronabedingt nicht im Präsenzformat stattfinden konnte.

Glücklicherweise konnte die Umplanung in einen Online-QZ erfolgreich mit tatkräftiger Umsetzung der Med Info Informationsmanagement im Gesundheitswesen $\mathrm{GmbH}$ umgesetzt werden. Der Referent wurde aus seinem häuslichen Büro bei Nürnberg zugeschaltet und rund 20 Pneumolog ${ }^{\star}$ Innen nahmen auf der virtuellen Plattform (Microsoft Teams) teil. Auffallend und sehr erfreulich war, dass zahlreiche Kolleg*Innen teilnahmen, die in den vergangenen Jahren nicht bei den QZ anwesend waren.

Damit wurde die Idee generiert, den QZPNW zukünftig als Hybrid anzubieten. Referent*Innen, wie auch Teilnehmer ${ }^{\star}$ Innen können somit entweder vor Ort präsent sein oder sich online zum QZ zuschalten und über die Plattform auch mitdiskutieren. Die genaue technische Umsetzung befindet sich derzeit auch in Bezug auf die Veranstaltungsräume in der Vorbereitungsphase, sollte aber keine unüberwindbaren Probleme bereiten. Die Organisatoren des QZPNW hoffen damit zukünftig dtl. mehr Kolleg* Innen als bisher im Rahmen der QZ live oder vir- tuell begrüßen zu können - im Idealfall alle von Böblingen bis Bad Mergentheim und von Heilbronn bis Aalen.

\section{Alexander Rupp Koordinator QZ Pneumologen Nordwürttemberg}

